\title{
La inclusión de personas con discapacidad en una escuela multideportiva: Efecto de las actitudes hacia la discapacidad en niños, niñas, jóvenes, padres, madres y personal de instrucción
}

\author{
Víquez Ulate, Fabián; Quirós Carrión, Sugey; Rodríguez-Méndez, Diego; Solano Mora, Luis \\ La inclusión de personas con discapacidad en una escuela multideportiva: Efecto de las actitudes hacia la \\ discapacidad en niños, niñas, jóvenes, padres, madres y personal de instrucción \\ MHSalud, vol. 17, núm. 2, 2020 \\ Universidad Nacional, Costa Rica \\ Disponible en: http://www.redalyc.org/articulo.oa?id=237062748002 \\ DOI: https://doi.org/10.15359/mhs.17-2.3
}

Esta obra está bajo una Licencia Creative Commons Atribución-NoComercial-SinDerivar 3.0 Internacional. 
La inclusión de personas con discapacidad en una escuela multideportiva: Efecto de las actitudes hacia la discapacidad en niños, niñas, jóvenes, padres, madres y personal de instrucción

The Inclusion of People with Disabilities in a Multi-Sport School: Effect of Attitudes on Children, Youth, Parents, and Instructors Towards Disability

A inclusão de pessoas com incapacidade numa escola poliesportiva: Efeitos das atitudes nas crianças, jovens, pais, mães e instrutores sobre a incapacidade

Fabián Viquez Ulate

Universidad Nacional, Costa Rica

fabian.viquez.ulate@una.cr

DOI: https://doi.org/10.15359/mhs.17-2.3

Redalyc: http://www.redalyc.org/articulo.oa?

(DD http://orcid.org/0000-0003-4980-3464

Sugey Quirós Carrión

Universidad Nacional, Costa Rica

suquiros12@gmail.com

(D) http://orcid.org/0000-0002-0976-3317

Diego Rodríguez-Méndez

Universidad Nacional, Costa Rica

diego.rodriguez.mendez@una.cr

(iD http://orcid.org/0000-0003-3164-539X

\section{Luis Solano Mora}

Universidad Nacional, Costa Rica

1solano@una.cr

(D) http://orcid.org/0000-0002-1556-6504 $\mathrm{id}=237062748002$

Recepción: 08 Julio 2019

Aprobación: 15 Abril 2020

\section{Resumen:}

El propósito del estudio fue observar el efecto de un programa multideportivo inclusivo sobre las actitudes de niños, niñas, jóvenes, padres, madres y personal de instrucción hacia las personas con discapacidad. Metodología: 18 niños, 4 instructores y 11 padres ( $\mathrm{n}=33$ ), pertenecientes a un programa multideportivo (EDP) de la Escuela de Movimiento Humano y Calidad de Vida, de la Universidad Nacional. Los actores fueron expuestos a una intervención que consistió en la participación en sesiones multideportivo, al menos 2 horas a la semana de 3 disciplinas deportivas (disciplinas individuales y de conjunto) que variaban semana a semana para un total de 6 semanas. Se les evaluó el cuestionario de "Actitudes hacia las personas con discapacidad" previo y posterior a la intervención. Los resultados fueron explorados mediante un análisis de varianza mixto3(grupo)x2(medición) con un alfa establecido en $\mathrm{p}<0.05$. Resultados: Se encontró una interacción significativa en la dimensión "valoración de las capacidades y limitaciones" (VCL), $\mathrm{F}(2,30)=10.28, \mathrm{p}<0.001$ y diferencias significativas en la vía mediciones en la dimensión de "clasificación genérica" $(\mathrm{CG}), \mathrm{F}(1,30)=4.884, \mathrm{p}=0.035$, mientras que en las dimensiones de "reconocimiento o negación de derechos" (RND) e "implicación personal" (IP) no se encontraron diferencias significativas ( $p>0.05)$. Conclusiones: La inmersión de diferentes actores en un programa multideportivo inclusivo ha sido eficaz al modificar positivamente algunas dimensiones (VCL y CG) de la actitud hacia las personas con discapacidad. Otras dimensiones que requieren una mayor interacción y vínculo con este tipo de poblaciones (RND e IP) probablemente necesiten una intervención más prolongada.

Palabras ClaVe: Cuestionario, integración social, intervención, deporte, discriminación.

\section{Abstract:}


This study aimed to observe children, youth, parents, and instructors' attitudes towards disability. The study intended to measure the effects of a multidisciplinary program of the Escuela Deportiva Pedagógica -EDP- (Pedagogical Sports School) of the School of Human Movement and Quality of Life, at the National University of Costa Rica. Methodology: thirty-three participants were incorporated into the study. Then, they were separated into three groups: four instructors, eleven parents, and eighteen children. The intervention consisted of participation in multisport sessions, at least two hours per week in three sport disciplines that varied from week to week over six weeks. All of them were given a questionnaire titled "Actitudes hacia las personas con discapacidad" (Attitudes towards people with disabilities). The questionnaire was applied both at the beginning and at the end of the intervention. A two-factor mixed ANOVA of 3 (groups) x 2 (measurements) with an alpha of 0.05 was used. Results: A significant interaction was found after the treatment in the dimension of "Valoración de las capacidades y limitaciones" -VCL(Assessment of skills and limitations), $\mathrm{F}(2,30)=10.28, \mathrm{p}<0.001$. There were also significant differences between measurements in the dimension of "Clasificación genérica" -CG- (generic classification), $F(1,30)=4.884, \mathrm{p}=0.035$, while in the dimensions of "Reconocimiento o negación de derechos" -RND- (recognition or denial of rights) and the "Implicación personal" -IP- (personal involvement) no significant differences were found (all $\mathrm{p}>0.05$ ). Conclusion: The results showed that the multidisciplinary programs of an inclusive nature positively modify attitudes (VCL and CG) towards the population with disabilities, but it is likely that some aspects related to attitude take longer to be changed (RND and IP).

KEYWORDS: questionnaire, social integration, intervention, sports, discrimination.

\section{Resumo:}

O objetivo do estudo foi observar o efeito de um programa poliesportivo inclusivo nas atitudes das crianças, jovens, pais, mães e instrutores em relação às pessoas com incapacidade. Metodologia: 18 crianças, 4 instrutores e 11 casais $(n=33)$ pertencentes a um programa poliesportivo (EDP) da Escola de Movimento Humano e Qualidade de Vida, da Universidade Nacional. Os atores foram expostos a uma intervenção que consistia em participar de sessões poliesportivas, pelo menos 2 horas por semana em 3 disciplinas esportivas (disciplinas individuais e em grupo) que variavam de semana para semana, durante um total de 6 semanas. O questionário "Atitudes em relação às pessoas com deficiência" foi avaliado antes e após a intervenção. Os resultados foram explorados por meio de uma análise mista de variância mista 3 (grupo) x 2 (medicao) com alfa estabelecido em p $<0,05$. Resultados: Foi encontrada interação significativa na dimensão Avaliação de capacidades e limitações (VCL), F $(2,30)=10,28, \mathrm{p}<0,001$ e diferenças significativas na via de medidas na dimensão Classificação Genérica $(G C), F(1,30)=4.884, p=0,035$, enquanto nas dimensões de reconhecimento ou negação de direitos (RND) e envolvimento pessoal (PI) não foram encontradas diferenças significativas $(p>0,05)$. Conclusões: A imersão de diferentes atores em um programa poliesportivo inclusivo tem sido eficaz na modificação positiva de algumas dimensões (VCL e GC) da atitude em relação às pessoas com incapacidade. Outras dimensões que requerem maior interação e vinculação com esse tipo de população (RND e IP) provavelmente requerem uma intervenção mais longa.

Palavras-Chave: questionário, integração social, intervenção, esporte, discriminação.

\section{INTRODUCCIÓN}

A nivel mundial se revela que el quince por ciento de la población posee alguna discapacidad. Desde esta aseveración, se plantea la necesidad de una sociedad más inclusiva y que comprenda, de una mejor manera, la discapacidad, para propiciar el cambio de actitudes hacia este grupo social, que ya de por sí, son personas prejuiciadas (Alcedo et ál., 2013; Galván y García, 2017; Organización Mundial de la Salud [OMS] y Banco Mundial [BM], 2011).

Ese prejuicio o actitud negativa probablemente se originó de los modelos biológicos donde las personas con discapacidad eran observadas como objetos de intervención, tratamiento y rehabilitación, los cuales se centraban en los déficits existentes en la periud; sin embargo, modelos más recientes parten de que la discapacidad es más de carácter social, donde el problema reside en las limitaciones impuestas por actitudes, posturas sociales, culturales y económicas de la población, que impiden su inserción en la sociedad y cercenan la participación plena y el disfrute de derechos en igualdad de condiciones (Galván y García, 2017; Hüg, Martos, Biassoni, Batista y Torres, 2019; Santana y Garoz, 2013).

Aún hoy, las personas con discapacidad suelen ser objeto de estereotipos negativos vinculados a nociones de anormalidad y falsas creencias. Se le atribuye a este colectivo la etiqueta de dependientes, inferiores, antisociales, incapaces de participar en la sociedad y emocionalmente inestables. De esta manera, las actitudes 
MHSALUd, ISSN: 1659-097X, 17(2), Julio-Diciembre, 2020, pP 1-13 Víguez Ulate, Quirós Carrión, Rodríguez-Méndez, Solano Mora

negativas se originan como uno de los principales obstáculos para la inclusión (Jenaro, Flores, Beltrán, Tomşa y Ruiz, 2016; Rello, Puerta y González, 2018; Rodríguez-Martín y Álvarez-Arregui, 2015).

En este sentido, las actitudes se pueden definir como una predisposición aprendida que ayuda a responder de cierta forma ante objetos, personas, grupos de personas o situaciones; éstas influyen en el tipo y la calidad de la interacción, de este modo, dependiendo de la actitud existente hacia las personas con discapacidad, así será la tendencia para comportarse hacia ellas (González-Hernández y Cortés-Arboleda, 2016; Hüg et ál., 2019; Rello, Puerta y González, 2018).

Se considera que las actitudes son una de las variables que más afecta en el momento de proporcionar u obstaculizar el proceso de inclusión para las personas con discapacidad en los diferentes ámbitos. De ahí la importancia de originar propuestas que las abordajes y se orienten a promover el reconocimiento y la aceptación de las diferencias, para alejar actitudes negativas hacia las personas con discapacidad, las cuales son identificadas como elementos de exclusión y base de actos de discriminación (Hüg et ál., 2019; Jenaro et ál., 2016; Pérez-Tejero, Ocete, Ortega-Vila y Coterón, 2012; Santana y Garoz, 2013).

La necesidad de cambiar dichas actitudes lleva a pensar que las intervenciones se deben dar de forma temprana, ya que éstas se aprenden especialmente en los primeros años de vida, periodo donde están menos arraigadas y en donde la educación, por medio de programas de sensibilización, favorece cambios positivos en las actitudes hacia la discapacidad (Novo-Corti, Muñoz-Cantero y Calvo-Porral, 2011; Pérez-Tejero et ál., 2012; Rello, Puerta y González, 2018; Rodríguez et ál.,2013; Santana y Garoz, 2013).

Es reiterado por varios estudios (Alcedo et ál., 2013; Felipe-Rello, Garoz y Tejero-González, 2020; Ocete, Pérez-Tejero y Coterón, 2015; Pérez-Tejero et ál., 2012) que integrar a la población con discapacidad depende de las actitudes de sus pares y docentes, más que del diseño de un currículo bien adaptado, además, el éxito de programas inclusivos está directamente relacionado con las actitudes positivas del lugar donde se implemente.

En este sentido, una de las teorías más utilizadas para lograr el cambio de actitudes e inclusión se basa en la teoría de contacto propuesta por Allport, Clark y Pettigrew (1954), citados por Castro (2006) y señala que el contacto proporciona un aumento de la comunicación y una reducción del prejuicio hacia el grupo minoritario, logra causar cambios de actitud como resultado de una recategorización de los diferentes grupos implicados, donde una situación de contacto óptimo introduce una identidad social o grupo social más amplio, o una supra entidad, la cual absorbe los diferentes grupos sociales participantes de la interacción, y reorienta los procesos cognitivos y motivacionales que usualmente se activan en los encuentros intergrupales.

Ahora bien, el contacto por sí solo no resulta efectivo, ya que son necesarios otros factores que afectan la interacción, tales como frecuencia, calidad, variedad, ámbitos y la atmósfera que lo rodea, además de los roles, el estatus y las características de los participantes en la interacción. Específicamente, se identificaron cuatro condiciones necesarias para la estructuración de un contacto intergrupal exitoso: a) la igualdad de estatus de sus participantes, b) la consecución de objetivos comunes, c) la cooperación intergrupal y d) el apoyo institucional (García, Díaz, Rodríguez, 2009; Pérez-Tejero et ál., 2012; Rello, Puerta y González, 2018).

De este modo, el contacto entre personas con discapacidad o sin ésta, en una situación de práctica deportiva cooperativa, conlleva a una mejora de las actitudes y se puede dar en tres contextos: a) el educativo, b) el deportivo, y c) el recreativo/social. Además debe tener en cuenta aspectos cuantitativos del contacto (frecuencia, duración, número de personas); el estado del contacto (inferioridad, igualdad, superioridad); rol dentro del contacto (competitivo o cooperativo); contexto social (real o artificial o voluntario u obligatorio); experiencias individuales (contacto previo con personas con discapacidad); ámbito del contacto (educativo, recreativo). Lo anterior debe caracterizarse por promover el logro de objetivos comunes; Asimismo, el respeto de las diferencias individuales debe ser gratificante y placentero (Pérez-Tejero et ál., 2012).

Las intervenciones en materia de movimiento humano respecto a las actitudes hacia la discapacidad han propiciado diversos escenarios a nivel escolar; por ejemplo, en primaria, el estudio de Felipe-Rello y GarozPuerta, (2014) manifestó que el $84 \%$ de los estudios analizados utilizan el contacto directo con las personas 
con discapacidad, con efectos positivos en cuanto a la intervención y durabilidad de ésta. En la misma línea, la investigación de Aguado, Alcedo y Arias (2008) indicó que el grupo que tuvo contacto directo con las personas con discapacidad mostró una actitud significativamente mejor hacia éstas que el grupo que no recibió el tratamiento. Por otra parte, el estudio de Santana y Garoz (2013) con adolescentes de secundaria evidenció un cambio de actitud hacia la discapacidad de forma significativa, por medio del contacto directo y de deportes adaptados; asimismo, el estudio de Polo-Sánchez y López-Justicia (2006), con estudiantado universitario, reveló que éste posee actitudes positivas hacia las personas con discapacidad, debido a que valoran su competencia. Por ello se puede afirmar que un modelo de intervención, donde exista la práctica deportiva inclusiva, va a ser un diseño completo y eficaz para modificar positivamente las actitudes hacia las personas con discapacidad, ya que fomenta la amistad, la cooperación y la ayuda entre grupos de iguales y desiguales (Felipe-Rello, Garoz y Tejero-González, 2020; Pérez-Tejero et ál., 2012; Rello, Puerta y González, 2018; Galván y García, 2017), de ahí la importancia de contar con espacios educativos que permitan la integración de las personas con discapacidad.

En este caso, las escuelas de iniciación deportiva comúnmente son unideportivas, especializadas en un deporte específico; con la modalidad del enfoque multideportivo, en la cual estas escuelas son un espacio donde las destrezas motoras y las habilidades deportivas se pueden potenciar en edades tempranas (Avella, Maldonado y Ramos, 2015; González, García, Contreras y Sánchez, 2009; Tabernero, Márquez y Llanos, 2001). En el ámbito costarricense existen programas y proyectos que atienden a las personas con discapacidad para el mejoramiento de su calidad de vida, la salud y el deporte, como, por ejemplo, Natación para Personas con Discapacidad Intelectual (NADI) y el programa Actividad Física Adaptada, Salud y Discapacidad (AFISADIS) (Natación para Personas con Discapacidad Intelectual [NADI], 2014; Actividad Física Adaptada, Salud y Discapacidad [AFISADIS], 2011).

En el ámbito costarricense, Monge y Monge (2009) afirman que existen necesidades educativas especiales en la clase de educación física, por lo cual se debe promover la inclusión; además, realizan una propuesta de actividades y reiteran la carencia de investigaciones en el área. En segunda instancia, respecto a la perspectiva de actitudes hacia la discapacidad en docentes universitarios costarricenses, un estudio de Soto (2007) evidencia que el $75 \%$ de docentes tiene una opinión neutral y el otro $25 \%$ una opinión negativa sobre el estudiantado que ingresa a la universidad. Mientras, Sanhueza, Granada y Bravo (2012) realizan una comparativa entre docentes universitarios de Costa Rica y de Chile y encuentran, en ambos cuerpos docentes, una actitud hacia la inclusión educativa positiva; manifiestan, también, que los recursos materiales y el tiempo constituyen una limitante para la realización de las clases. A nivel motriz, Castro (2010) evidencia la implementación de actividades recreativas en personas con necesidades educativas especiales y madres para reducir los niveles de estrés, depresión y ansiedad. Otro estudio de Madrigal y Solano, (2008) determina la capacidad aeróbica y la composición corporal en personas con retraso mental y síndrome de Down; por último, Hernández (2018) aporta la influencia del entrenamiento sensorial en el equilibrio estático en nadadores con síndrome de Down.

La evidencia anterior, confirma investigaciones en áreas referidas a temas de discapacidad, pero no se visualiza la integración de padres, madres, instructores, infantes, jóvenes y personas con discapacidad en el ámbito deportivo de formación inicial en una escuela multideportiva, ni el posible impacto positivo que podrían brindar los programas desde el contexto escolar hacia la discapacidad.

\section{Metodología}

El presente estudio es de tipo cuasiexperimental, ya que busca adecuar el diseño de investigación al ámbito real, trata de controlar algunas variables que puedan afectar la validez interna del estudio (Thomas, Nelson, y Silverman, 2015). En este caso en particular, por sus características, el estudio no presentó grupo control ni aleatorización, pues ya era un grupo conformado. 
MHSALUd, ISSN: 1659-097X, 17(2), Julio-Diciembre, 2020, pP 1-13 Víguez Ulate, Quirós Carrión, Rodríguez-Méndez, Solano Mora

\section{Participantes}

Participaron 33 personas, a saber: a) 11 padres y madres ( $X=41.5 \pm 10.5$ años), b) 4 instructores $(X=21$ \pm 3.4 años), c) 18 personas usuarias sin discapacidad intelectual ni física $(X=10.3 \pm 2.5$ años). De eéstos, 15 son infantes y 3 adolescentes en edad colegial.

Cabe agregar que también participaron 4 niñas y 2 niños con discapacidad ( $\mathrm{X}=11 \pm 2.7)$; 2 con discapacidades físicas, 3 con discapacidad cognitiva y 1 con discapacidad múltiple. Si bien, este grupo no fue evaluado en el estudio, al ser esta una investigación inclusiva, se especifica por ser una parte medular de la investigación.

Los criterios de inclusión en el estudio fueron: pertenecer al proyecto Escuela Deportiva Pedagógica y poseer el consentimiento informado de estudiantes de la universidad, padres y madres, además de la aprobación del grupo de menores de edad.

\section{Instrumentos y materiales}

Se utilizaron los siguientes documentos para la recolección de información: carta de consentimiento informado por parte del estudiantado universitario, padres y madres, además de la carta de asentimiento para cada menor de edad.

Asimismo, se utilizó la escala de Actitudes hacia las personas con discapacidad (Verdugo, Jenaro y Arias, 1995). Se trata de una escala multidimensional desarrollada en España, que cuenta con estudios de fiabilidad $(\alpha$ de Cronbach $=.92)$ y validez por criterio experto. Esta misma ha sido validada en niños y niñas entre los 7 y los 10 años, con resultados de alfas de Cronbach altos en las distintas dimensiones que variaban entre los 0.767 y 0.864 (Alcedo et ál., 2013). Además, se reportaron valores de alfas de Cronbach entre 0.84 y 0.92 para estudiantes de colegio y universidad (Polo-Sánchez y López-Justicia, 2006; Suriá-Martínez, 2011) y un alfa de Cronbach de 0.85 en personas mayores (Barradas-Alarcón, Robledo-Salinas, Guzmán-Ibáñez, GutiérrezSerrano y Sánchez-Barradas, 2014).

Está formada por 37 ítems, algunos de los cuales expresan valoración negativa $(1,2,4,5,6,7,8,10,15,17$, 18, 23, 24, 25, 26, 28, 29, 31, 34, 35 y 37). Las dimensiones del instrumento son las siguientes: a) Valoración de las capaiudades y limitaciones (VCL) (reactivos 1, 2, 4, 7, 8, 16, 21, 29 y 36); b) Reconocimiento o negación (RND) 5 (reactivos 6, 9, 12, 13, 14, 15, 22, 23, 27, 35 y 37); c) Implicación personal (IP) (reactivos 3, 5, 10, 11, 25, 26 y 31); d) Clasificación genérica (CG) (reactivos 18, 24, 28 y 34); e) Asunción de roles (AR) (reactivos 19, 30 y 33) (Verdugo, Jenaro y Arias, 1995).

La escala es de tipo Likert de 1 a 6 , los significados de las opiniones son los siguientes: 1.- Estoy muy de acuerdo (MA); 2.- Estoy bastante de acuerdo (BA); 3.- Estoy parcialmente de acuerdo (PA); 4.- Estoy parcialmente en desacuerdo (PD); 5.- Estoy bastante en desacuerdo (BD); 6.- Estoy en total desacuerdo (TD) y evalúa las actitudes ante personas con cualquier tipo de discapacidad. De este modo, una puntuación próxima a seis para cualquier ítem de la escala refleja una actitud positiva ante las personas con discapacidad. Así, la persona evaluada debe opinar si está de acuerdo o no con cada una de las frases que se presentan, formuladas positiva o negativamente (Alcedo et ál., 2013).

\section{Procedimiento}

El estudio se realizó en la Universidad Nacional, en la Escuela Ciencias del Movimiento Humano y Calidad de Vida, en el proyecto de la Escuela Deportiva Pedagógica (EDP), la cual se basa en la práctica de multideportes una vez por semana, en un lapso de cuatro horas, y atiende población entre los 7 a 14 años. 
Se analizó la viabilidad del estudio; se solicitaron los permisos respectivos; se definieron las actividades, horarios, población, capacitaciones, instrumentos y colaboradores que participaron en la investigación.

Se convocó al grupo de participantes para explicar en qué consistía el estudio; a quienes aceptaron se les pasó el consentimiento y asentimiento informado, junto con el cuestionario Actitudes hacia las personas con discapacidad (pretest). El protocolo de investigación contó con los permisos de las entidades respectivas, además se implementaron todas las normas del protocolo de Helsinki. Posteriormente se capacitó al equipo instructor sobre los procesos inclusivos en el deporte adaptado y la recreación para la población con discapacidad participante.

Se incorporó, en igualdad de condiciones, a las personas con discapacidad a las sesiones deportivas y recreativas, las cuales consistían en el aprendizaje técnico de los diferentes deportes, participación colectiva en éstos; actividades recreativas grupales; además, se efectuó una charla sobre discapacidad para los padres y madres participantes del proyecto. La intervención consistió en seis semanas de actividades deportivas y juegos recreativos, al menos 2 horas a la semana en 3 disciplinas deportivas que variaban semana a semana; después de las seis semanas se procedió a aplicar el postest. (En agradecimiento a las personas participantes se les dio la oportunidad de seguir en el proyecto de forma gratuita). En última instancia se llevó a cabo la tabulación de los datos y su respectivo análisis estadístico.

Cabe mencionar que ninguno de los participantes en el estudio tuvo contacto previo con alguna persona con discapacidad, en el marco de actividades deportivas-recreativas, exceptuando algunos instructores, padres y madres que sí habían tenido contacto, pero sin una interacción directa.

\section{Análisis estadístico}

Se calcularon las estadísticas descriptivas para las variables estudiadas y, posteriormente, se realizó un ANOVA de 2 vías mixta 3 (grupos) x 2 (mediciones); en caso de diferencias significativas en los grupos se decidió aplicar el post hoc de Bonferroni. Todo esto se hizo mediante el paquete estadístico SPSS versión 24.0 para Windows. El alfa fijada en este estudio fue de $5 \%$.

\section{Resultados}

Para analizar la asunción de la normalidad de los datos, se aplicaron las pruebas estadísticas por gráficos

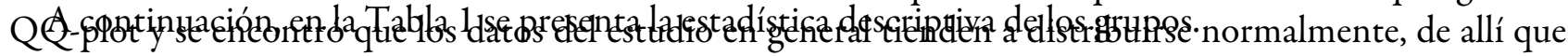
se pudiera aplicar estadística paramétrica. 
MHSALUd, ISSN: 1659-097X, 17(2), Julio-Diciembre, 2020, pP 1-13

Víguez Ulate, Quirós Carrión, Rodríguez-Méndez, Solano Mora

TABLA 1

Estadistica descriptiva del estudio

\begin{tabular}{lccc}
\hline Grupos & $\mathrm{N}$ & Sexo & Edad X (DS) \\
\hline $\begin{array}{l}\text { Instructores } \\
\begin{array}{l}\text { Padres y } \\
\text { madres }\end{array}\end{array}$ & 4 & M: 2, F: 2 & $21 \pm 3,4$ \\
$\begin{array}{l}\text { Personas } \\
\begin{array}{l}\text { usuarias sin } \\
\text { discapacidad }\end{array}\end{array}$ & 11 & M: 15, F: 3 & $10,3 \pm 2,5$ \\
\hline
\end{tabular}

Nota: M: masculino, F: femenino.

Con respecto a la ANOVA de 2 vías mixto, los análisis mostraron que hubo interacción significativa en la valoración de las capacidades y limitaciones (VCL), F(2,30) $=10.28, \mathrm{p}<0.001$. Al realizar los análisis de efectos simples, se halló que las mediciones fueron las que generaron la interacción significativa $(\mathrm{p}<0.017)$, y se encontró que la VCL mejoró después de las 6 semanas.

En el caso del reconocimiento o negación de derechos (RND), el ANOVA de 2 vías mixto mostró que no hubo interacción estadísticamente significativa, $\mathrm{F}(2,30)=0.873, \mathrm{p}=0.428$; tampoco hubo diferencias significativas en las mediciones $\mathrm{F}(1,30)=2.68, \mathrm{p}=0.112$, ni entre grupos $\mathrm{F}(2,30)=1.53, \mathrm{p}=0.233$.

Para la implicación personal (IP), el ANOVA de 2 vías mixto no evidenció interacciones estadísticamente significativas $\mathrm{F}(2,30)=0.651, \mathrm{p}=0.529$, ni diferencias significativas en las mediciones $\mathrm{F}(1,30)=2.473$, $\mathrm{p}=0.126$, mientras que sí mostró diferencias significativas entre los grupos $\mathrm{F}(2,30)=3.395, \mathrm{p}=0.047$. El análisis post hoc DMS estableció que los padres y madres tenían un IP significativamente más alto que los usuarios sin discapacidad $(\mathrm{p}=0.034)$.

En la clasificación genérica (CG), el ANOVA de 2 vías mixto no indicó una interacción significativa $\mathrm{F}(2$, $30)=1.419, \mathrm{p}=0.258$, ni diferencias significativas en la $\mathrm{CG}$ de los distintos grupos $\mathrm{F}(2,30)=0.731, \mathrm{p}=$ 0.490 , pero sí mostró diferencias significativas en las mediciones $F(1,30)=4.884, p=0.035$, y encontró que al final de las 6 semanas aumentó, en general, la CG.

Finalmente, el ANOVA de 2 vías mixto para la asunción de roles (AR) no determinó una interacción significativa $\mathrm{F}(2,30)=2.748, \mathrm{p}=0.080$, ni diferencias significativas en las mediciones $\mathrm{F}(1,30)=2.582, \mathrm{p}=$ 0.119 , ni entre los grupos $\mathrm{F}(2,30)=1.701, \mathrm{p}=0.200$ (Ver Tabla 2). 
MHSALUD, ISSN: 1659-097X, 17(2), JULIO-DiCIEMBRE, 2020, PP 1-13

Víouez Ulate, Quirós Carrión, Rodríguez-Méndez, Solano Mora

TABLA 2

Promedios pre y post, junto con el porcentaje de cambio por grupo en cada dimensión

\begin{tabular}{ccccccccccc}
\hline Dimensión & \multicolumn{2}{c}{$\begin{array}{c}\text { Usuarios sin } \\
\text { discapacidad }\end{array}$} & \multicolumn{3}{c}{ Instructores } & \multicolumn{5}{c}{ Padres y madres } \\
\hline & Pre & Post & $\begin{array}{c}\text { \% } \\
\text { cambio }\end{array}$ & Pre & Post & $\begin{array}{c}\text { \% } \\
\text { cambio }\end{array}$ & Pre & Post & $\begin{array}{c}\text { \% } \\
\text { cambio }\end{array}$ & Total \\
VCL & 62,10 & 85,19 & 37,18 & 80,00 & 89,86 & 12,33 & 75,76 & 69,70 & $-8,00$ & 17,10 \\
RND & 79,61 & 89,35 & 12,33 & 91,67 & 95,83 & 4,55 & 81,82 & 83,94 & 2,59 & 7,97 \\
IP & 76,19 & 86,19 & 13,13 & 92,14 & 93,57 & 1,55 & 88,31 & 92,99 & 5,30 & 8,75 \\
CG & 71,11 & 85,78 & 20,63 & 70,00 & 79,00 & 12,86 & 70,55 & 73,09 & 3,61 & 14,04 \\
AR & 86,11 & 66,67 & $-22,58$ & 87,50 & 80,00 & $-8,57$ & 68,18 & 70,45 & 3,33 & $-13,40$ \\
\hline
\end{tabular}

\section{Discusión}

En cuanto a los resultados obtenidos en el estudio, se observaron diferencias significativas entre mediciones en las dimensiones VCL y la CG, lo cual concuerda con estudios como el de González y Baños (2012); Lara y Ortiz (2011); Molina y Valenciano (2014) y Pérez-Tejero et ál. (2012), quienes también reportaron cambios positivos en esas variables. Con ello se reafirma la teoría de que el contacto con personas con alguna discapacidad ayuda a modificar las actitudes hacia este colectivo, siempre y cuando esta sea planificada y frecuente (García y Hernández, 2011; García, Díaz y Rodríguez, 2009; Novo-Corti, Muñoz-Cantero y Calvo-Porral, 2011; Sánchez, 2017). Otros estudios encontraron hallazgos similares; reportan mejoras a nivel de actitudes cuando se utiliza el contacto con personas con discapacidad en ambientes deportivos recreativos, por lo cual estas intervenciones constituyen una herramienta normalizadora y de inclusión social inigualable (Abellán et ál., 2018; Contreras y Abellán, 2018; González y Baños, 2012; Ocete, Pérez-Tejero y Coterón, 2015; Rello, Puerta y González, 2018). En este sentido, se puede establecer que la percepción sobre las capacidades y limitaciones del colectivo con discapacidad varía en forma positiva, es decir, se creen más capaces de realizar diferentes actividades.

Para las otras dimensiones analizadas (RND, IP y AR), no se mostraron cambios significativos del pretest al postest, esto pudo deberse a distintas situaciones; la primera es que la muestra no fue lo suficientemente grande, lo que pudo haber afectado la significancia de los resultados; por otra parte, el tiempo de intervención posiblemente no tuvo la suficiente duración total ni frecuencia de veces por semana para poder determinar cambios significativos en estas dimensiones. Hernández y Martínez (2016) y Santana y Garoz (2013) habían recomendado un mayor volumen de contacto con las personas con una discapacidad; del mismo modo, el que las personas sin discapacidad tuvieran un mayor seguimiento educativo en temáticas relacionadas con la discapacidad permitía mejorar su actitud hacia la discapacidad.

Ahora bien, aunque se indicó que las dimensiones anteriores no presentaron diferencias significativas, sí se observó que el porcentaje de cambio analizado en las dimensiones RND e IP mostró una tendencia a mejorar. Esto brinda indicios de que se debe tener mayor tiempo de contacto con la población con discapacidad para conseguir cambios positivos en la percepción y forma de relacionarse con ésta. Datos similares se encontraron en estudios como el de Abellán, Sáez-Gallego y Reina (2018) y Felipe-Rello y Garoz-Puerta (2014). En el caso de la dimensión $\mathrm{AR}$, esta se comportó totalmente diferente a los resultados de las otras dimensiones: muestra una disminución en su porcentaje de cambio, lo cual puede explicarse debido a que la técnica del contacto mejora las actitudes hacia las personas con discapacidad y el conocimiento sobre este tema, pero 
MHSALUd, ISSN: 1659-097X, 17(2), Julio-Diciembre, 2020, pP 1-13 Víguez Ulate, Quirós Carrión, Rodríguez-Méndez, Solano Mora

no consigue establecer vínculos más intensos en cuanto al cómo se considera que se siente la otra persona (Rodríguez et ál., 2013; Slininger, Sherrill, Jankowski, 2000). A futuro, será necesario detallar intervenciones con una mayor participación de los padres y madres o que estén específicamente dirigidas a estos, para poder generar un mayor impacto en el cambio de esta variable.

Por último, se observó que los porcentajes de cambio más altos se dieron en el grupo de usuarios sin discapacidad e instructores, ya que estos grupos fueron los que mantuvieron mayor contacto directo con las personas con discapacidad, mientras que los padres y madres obtuvieron los porcentajes de cambio más bajos, pues no interactuaron al mismo nivel que los grupos anteriores. Esta observación afirma la idea de que la cantidad y calidad del contacto es básico para cambiar la actitud hacia la discapacidad (Abellán, Sáez-Gallego y Reina, 2018; Felipe-Rello y Garoz-Puerta, 2014; Pérez-Tejero et ál., 2012; Santana y Garoz, 2013).

En lo concerniente a las diferencias en la dimensión IP entre los grupos padres y madres e usuarios sin discapacidad, esto pudo deberse a que las personas con mayor edad y mayor grado de escolaridad tienen mejor actitud hacia la discapacidad que las personas jóvenes y con menor escolaridad (Lara y Ortiz, 2011; Martínez y Bilbao, 2013).

\section{CONCLUSIÓN}

Según los resultados del presente estudio, se puede concluir que los programas multideportes de carácter inclusivo ayudan, en forma general, a modificar las actitudes sobre la población con discapacidad, pero es probable que algunos aspectos relacionados con estas actitudes tarden más tiempo en ser transformadas que otras; lo que sí queda claro es que la magnitud de este cambio va a depender de la cantidad y la calidad del contacto entre las poblaciones.

De manera más específica, se evidenció en esta investigación que después de un contacto de seis semanas, las dimensiones: valoración de las capacidades y limitaciones (VCL) y clasificación genérica (CG) fueron las más susceptibles a mejorar; además, los usuarios sin discapacidad e instructores tuvieron mayores cambios que el grupo de padres y madres, lo que respalda la teoría de que los espacios de interacción contribuyen a mejorar las actitudes hacia el colectivo con discapacidad.

Finalmente, se recomienda que este tipo de iniciativas se promuevan en edades tempranas en distintas instituciones, sean estas educativas públicas, privadas y deportivas, entre otras, con el objetivo de evitar actitudes predispuestas hacia las personas con discapacidad.

Del mismo modo, se recomienda ampliar el tiempo de contacto y la intensidad es éste, con la idea de verificar si existen mayores cambios en el ámbito de las actitudes y medir el periodo de retención de estas nuevas actitudes.

También, es importante acompañar el proceso con espacios de capacitación a instructores y demás actores educativos, con el propósito de favorecer la sensibilización. Estos espacios deben darse en una forma periódica a todas las personas implicadas en el proceso.

\section{ReFERENCIAS}

Abellán, J., Sáez-Gallego, N. y Reina, R. (2018). Explorando el efecto del contacto y el deporte inclusivo en educación física en las actitudes hacia la discapacidad intelectual en estudiantes de secundaria. RICYDE. Revista Internacional de Ciencias del Deporte, 14(53), 233-242. doi: https://doi.org/10.5232/ricyde2018.05304

Actividad Física Adaptada, Salud y Discapacidad [AFISADIS]. (2011). Actividad física adaptada, salud y discapacidad. https://www.ciemhcavi.una.ac.cr/index.php/afisadis

Aguado, A., Alcedo, M., y Arias, B. (2008). Cambio de actitudes hacia la discapacidad con escolares de primaria. Revista Psicothema, 20(4), 697-704.https://sid.usal.es/idocs/F8/ART11149/cambio_actitudes_discapacidad_escolar es_primaria.pdf 
MHSALUD, ISSN: 1659-097X, 17(2), JULIO-DiCIEMBRE, 2020, PP 1-13

Víouez Ulate, Quirós Carrión, Rodríguez-Méndez, Solano Mora

Alcedo, M. A., Gómez, L. E., Aguado, A. L., Arias, B. y González, R. (2013). Eficacia del contacto e información como técnicas de cambio de actitudes hacia personas con discapacidad en niños de Educación Primaria. Universitas Psychologica, 12(2), 493-50 4. http://www.scielo.org.co/pdf/rups/v12n2/v12n2a16.pdf

Allport, G. W., Clark, K., y Pettigrew, T. (1954). The nature of prejudice. $\quad$ althaschool.org/_cache/files/7/1/71f96bdb-d4c3-4514bae2-9bf809ba9edc/97F5FE75CF9A120E7DC108EB1B0FF5EC.holocaust-the-nature-of-prejudice.doc

Avella, R., Maldonado, C., y Ramos, S. (2015). Entrenamiento deportivo con niños. Kinesis.

Barradas-Alarcón, Robledo-Salinas, Guzmán-Ibáñez, Gutiérrez-Serrano y Sánchez-Barradas. (2014). Actitudes hacia las personas con discapacidad en académicos de la Universidad Veracruzana. Revista Iberoamericana de Producción Académica y Gestión Educativa, 1(2).

Castro, J. (2010). Implementación de módulos educativos de actividad física-recreativa en madres de niños y niñas con necesidades educativas especiales, como medios para reducir niveles de estrés, depresión y algias corporales [Tesis de maestría], Universidad Nacional, Heredia, Costa Rica].

Castro, V. (2006). La psicología social de las relaciones intergrupales: Modelos e hipótesis. Actualidades en psicología, 20(107), 45-71.http://pepsic.bvsalud.org/scielo.php?script=sci_arttext\&pid=S0258-64442006000100003\&l $\mathrm{ng}=\mathrm{pt} \& \mathrm{t} \operatorname{lng}=\mathrm{es}$

Contreras, M. y Abellán, J. (2018). Mejorando las actitudes hacia la discapacidad en Educación Física a través del deporte adaptado. E-motion: Revista de Educación, Motricidad e Investigación, 11, 3-15. http://rabida.uhu.es/d space/bitstream/handle/10272/15803/Mejorando.pdf?sequence $=2$

Felipe-Rello, C. y Garoz-Puerta, I. (2014). Actividad físico-deportiva en programas de cambio de actitudes hacia la discapacidad en edad escolar: Una revisión de la literatura. Cultura, Ciencia y Deporte, 9(27), 199-210. https: //doi.org/10.12800/ccd.v9i27.462

Felipe-Rello, C., Garoz, I. y Tejero-González, C. (2020). Cambiando las actitudes hacia la discapacidad: Diseño de un programa de sensibilización en Educación Física. Retos: Nuevas Tendencias En Educación Física, Deporte y Recreación, 37(1), 713-721. http://web.a.ebscohost.com.una.idm.oclc.org/ehost/pdfviewer/pdfviewer?vid=1 \&sid=b52f2fd2-16c8-4f0f-9c30-5700b06c2426\%40sessionmgr 4007

Galván, J. L, y García, I. (2017). Actitudes de los pares hacia niños y niñas en condición de discapacidad. Actualidades Investigativas en Educación, 17(2), 1-25. doi: http://dx.doi.org/10.15517/aie.v17i2.28673

García L. G, y Hernández O. S. (2011). Actitudes hacia la discapacidad de jóvenes y adultos de Chiapas. Universitas Psychologica, 10(3). https://www.redalyc.org/pdf/647/64722377014.pdf

García, M. A. F., Díaz, A. L. A., y Rodríguez, M. Á. A. (2009). Revisión y análisis de los programas de cambio de actitudes hacia personas con discapacidad. Annuary of Clinical and Health Psychology, 5, 85-98. http://instituc ional.us.es/apcs/doc/APCS_5_esp_85-98.pdf

González, J., y Baños, L. M. (2012). Estudio sobre el cambio de actitudes hacia la discapacidad en clases de actividad física. Cuadernos de Psicologia del Deporte, 12(2), 101-108. http://scielo.isciii.es/pdf/cpd/v12n2/articulo10.pdf

González-Hernández, J., y Cortés-Arboleda, M. R. (2016). Actitudes y creencias hacia la discapacidad en clases de educación física. Una cuestión educativa. Psychology, Society \& Education, 8(2), 105-120. https://dialnet.uniri oja.es/servlet/articulo?codigo $=6360230$

González, S., García, L., Contreras, O., y Sánchez, D. (2009). El concepto de iniciación deportiva en la actualidad. RETOS. Nuevas Tendencias en Educación Física, Deporte y Recreación, (15), 14-20. http://www.redalyc.org/pd f/3457/345732280003.pdf

Hernández, M. (2018). Influencia de un programa de entrenamiento sensoriomotor sobre el equilibrio estático en nadadores con sindrome de down y discapacidad intelectual [Tesis de maestría], Universidad Nacional, Heredia, Costa Rica.

Hernández, J. A., y Martínez, A. H. (2016). Los juegos motores sensibilizadores y su efecto en las actitudes hacia la discapacidad mostradas por futuros maestros de Educación Física. Sportis. Scientific Journal of School Sport,Physical Education and Psychomotricity, 2(1), 93-106. doi: https://doi.org/10.17979/sportis.2016.2.1.14 43 
MHSALUd, ISSN: 1659-097X, 17(2), Julio-Diciembre, 2020, pP 1-13 Víguez Ulate, Quirós Carrión, Rodríguez-Méndez, Solano Mora

Hüg, M., Martos, G., Biassoni, P., Batista, L., \& Torres, E. (2019). Actitudes hacia la discapacidad en adolescentes: Efectos de un programa basado en el modelo social de discapacidad. Revista Latinoamericana en Discapacidad, Sociedad y Derechos Humanos, 3(1). http://redcdpd.net/revista/index.php/revista/article/view/148/0

Jenaro, C., Flores, N., Beltrán, M., Tomşa, R., y Ruiz, M. I. (2016). Necesidades educativas e inclusión escolar: El peso de las actitudes. International Journal of Developmental and Educational Psychology, 4(1), 605-612. http://infa d.eu/RevistaINFAD/wp-content/uploads/0214-9877_2014_1_4_605.pdf

Lara, G. A. G., y Ortiz, S. H. (2011). Actitudes hacia la discapacidad de jóvenes y adultos de Chiapas. Universitas Psychologica, 10(3), 817-827. http://www.scielo.org.co/pdf/rups/v10n3/v10n3a14.pdf

Madrigal, L. y Solano, H. (2008). Capacidad aeróbica y composición corporal en sujetos con retraso mental y sindrome de down [Tesis de maestría], Universidad Nacional, Heredia, Costa Rica].

Martínez, M., y Bilbao, L. (2013). Los docentes de la universidad de Burgos y su actitud hacia las personas con discapacidad. http://riberdis.cedd.net/bitstream/handle/11181/3770/Los\%20docentes\%20de\%20la\%20Univ ersidad\%20de\%20Burgos.pdf?sequence $=1$

Molina, J. P., y Valenciano, J. (2014). Creencias y actitudes hacia un profesor de educación física en silla de ruedas. Un estudio de caso. Revista de Psicología del Deporte, 19 (1), 136-149. https://www.redalyc.org/pdf/2351/235 116414009.pdf

Monge, M., y Monge, M. (2009). Cuerpos, mentes y aprendizajes diversos: La clase de Educación Física como modelo de una educación inclusiva en Costa Rica. Revista de Innovación Educativa, (9), 115-136. http://riberdis.cedd .net/handle/11181/3838

Natación para Personas con Discapacidad Intelectual [NADI]. (2014). Natación para personas con discapacidad intelectual. https://www.ciemhcavi.una.ac.cr/index.php/nadi

Novo-Corti, I., Muñoz-Cantero, J. M., y Calvo-Porral, C. (2011). Análisis de las actitudes de los jóvenes universitarios hacia la discapacidad: Un enfoque desde la teoría de la acción razonada. Relieve-Revista Electrónica de Investigación y Evaluación Educativa, 17(2). https://www.uv.es/RELIEVE/v17n2/RELIEVEv17n2_5.pdf

Ocete, C., Pérez-Tejero, J, Coterón, J. (2015). Propuesta de un programa de intervención educativa para facilitar la inclusión de alumnos con discapacidad en educación física. Retos: nuevas tendencias en educación física, deporte y recreación, 27, 140-145. https://dialnet.unirioja.es/servlet/articulo?codigo $=5407732$

Organización Mundial de la Salud y Banco Mundial. (2011). Informe mundial sobre la discapacidad. https://apps.wh o.int/iris/bitstream/handle/10665/75356/9789240688230_spa.pdf

Pérez-Tejero, J., Ocete, C., Ortega-Vila, G., y Coterón, J. (2012). Diseño y aplicación de un programa de intervención de práctica deportiva inclusiva y su efecto sobre la actitud hacia la discapacidad: El campus inclusivo de baloncesto. RICYDE. Revista Internacional de Ciencias del Deporte, 8(29), 258-271. https://dialnet.unirioja.es $/$ servlet/articulo?codigo $=4248516$

Polo-Sánchez, M., y López-Justicia, M. (2006). Actitudes hacia las personas con discapacidad de estudiantes de la Universidad de Granada. Revista Española de Orientación y Psicopedagogía, 17(2), 195-211. https://doi.org/1 0.5944/reop.vol.17.num.2.2006.11346

Rello, C. F., Puerta, I. G., y González, C. M. T. (2018). Análisis comparativo del efecto de tres programas de sensibilización hacia la discapacidad en Educación Física. Retos: nuevas tendencias en educación física, deporte y recreación, 34, 258-262. https://recyt.fecyt.es/index.php/retos/article/view/59889/38611

Rodríguez, A., Ángeles, M., Gómez, L. E., Aguado, A. L., Arias, B., y González, R. (2013). Eficacia del contacto e información como técnicas de cambio de actitudes hacia personas con discapacidad en niños de Educación Primaria. Universitas Psychologica. 12 (2), 493-504. http://www.scielo.org.co/pdf/rups/v12n2/v12n2a16.pdf

Rodríguez-Martín, A., y Álvarez-Arregui, E. (2015). Universidad y discapacidad: Actitudes del profesorado y de estudiantes. Perfiles educativos, 37(147), 86-102. http://www.scielo.org.mx/scielo.php?script=sci_arttext\&pid $=$ S0185-26982015000100006\&lng $=$ es\&tlng $=$ es.

Sánchez, M. R. (2017). Actitudes hacia la discapacidad en alumnos de Magisterio de Educación Infantil. Propuestas de formación para una educación inclusiva. Revista de Educación Inclusiva, 8(3). http://www.revistaeducacion inclusiva.es/index.php/REI/article/view/95/92 
Sanhueza, S., Granada, M., y Bravo, L. (2012). Actitudes del profesorado de Chile y Costa Rica hacia la inclusión educativa. Cadernos de Pesquisa, 42(147), 884-899. http://repositorio.minedu.gob.pe/bitstream/handle/1234 56789/944/2012_Henriquez_Actitudes del profesorado de chile y costa rica hacia la inclusión educativa_.pdf? sequence $=1 \&$ isAllowed $=\mathrm{y}$

Santana, P., y Garoz, I. (2013). Actitudes hacia la discapacidad e intervención docente desde el deporte adaptado. Journal of Medicine and Science of Physical Activity and Sport, 13, 1-17. http://www.redalyc.org/articulo.oa?id $=54225676001$

Soto, C. (2007). Actitud docentes de la Universidad de Costa Rica hacia los (as) estudiantes con discapacidad de la Universidad. Revista Educación, 31(1), 11-42. https://revistas.ucr.ac.cr/index.php/educacion/article/view/12 $51 / 1314$

Slininger, D., Sherrill, C., y Jankowski, C. M. (2000). Children's attitudes toward peers with severe disabilities: Revisiting contact theory. Adapted Physical Activity Quarterly, 17(2), 176-196. https://doi.org/10.1123/apaq. 17.2.176

Suriá-Martínez, R. (2011). Análisis comparativo sobre las actitudes de los estudiantes hacia sus compañeros con discapacidad.http://riberdis.cedd.net/xmlui/bitstream/handle/11181/3635/actitudes_estudiantes_companer os_discapacidad.pdf?sequence $=1$

Tabernero, B., Márquez, S., y Llanos, M. (2001). Elementos a analizar en el proceso de iniciación deportiva. Retos: nuevas tendencias en educación fisica, deporte y recreación, I (1). https://dialnet.unirioja.es/servlet/articulo?codi go $=2283333$

Thomas, J. R., Nelson, J. K., y Silverman, S. J. (2015). Research methods in physical activity (7 ed). Human Kinetics.

Verdugo, M. Á., Jenaro, C., y Arias, B. (1995). Actitudes sociales y profesionales hacia las personas con discapacidad: Estrategias de evaluación e intervención. En M. Á. Verdugo (Ed.), Personas con discapacidad: perspectivas psicopedagógicas y rehabilitadoras (pp. 79- 143). Siglo XXI.

\section{BY-NC-ND}

